\title{
Imaginaires archéologiques, MCC-SDARCHETIS
}

\section{Emmanuel Ghesquière}

\section{OpenEdition}

\section{Journals}

Édition électronique

URL : http://journals.openedition.org/rao/971

DOI : $10.4000 /$ rao 971

ISBN : 978-2-7535-1609-0

ISSN : 1775-3732

Éditeur

Presses universitaires de Rennes

Édition imprimée

Date de publication : 31 décembre 2009

ISBN : 978-2-7535-1086-9

ISSN : 0767-709x

\section{Référence électronique}

Emmanuel Ghesquière, «Imaginaires archéologiques, MCC-SDARCHETIS », Revue archéologique de I'Ouest [En ligne], 26 | 2009, mis en ligne le 31 décembre 2011, consulté le 04 décembre 2020. URL http://journals.openedition.org/rao/971 ; DOI : https://doi.org/10.4000/rao.971 
prend ici une dimension christique pour se métamorphoser de sculptures actuelles à témoins archéologiques.

L'article de Pierre Lagrange traite du délicat sujet de l'Atlantide, versé actuellement dans le mythe plus ou moins lié à l'âge du Bronze européen. Ici l'auteur traite de la période entre les deux guerres où son étude relevait encore du champ scientifique, où l'on s'interrogeait sur la possible présence quaternaire d'un continent dans l'Atlantique, reliant l'Europe ou l'Afrique aux Amériques. Il s'avère maintenant que ce continent a disparu depuis trop longtemps pour que l'humanité en ait gardé le souvenir, mais dans les années 1920, atlantéens et atlantologues se sont opposés dans leur recher- che sur le continent disparu, dérivant finalement sur les divagations mystiques qui perdurent encore actuellement.

Louvrage s'achève sur une contribution de Gaétano Ciarcia. Il y traite des travaux de l'ethnologue Marcel Griaule en Afrique, traquant les civilisations disparues par un mélange d'ethnologie et d'archéologie et aménageant les résultats suivant sa convenance.

En bref, un livre offrant une lecture différente de l'archéologie, nous renvoyant parfois sa pratique dans un miroir déformé, loin de la discipline scientifique que nous pratiquons. Quoique...

Emmanuel GHESQUiÈRE INRAP Basse-Normandie et UMR 6566-CReAAH

Chouquer, G., 2008 - Traité d'archéogéographie. La crise des récits géohistoriques, Paris, Errance, 199 p. (ISBN 978-2-87772$374-9 ; 24 €)$.

Il est ardu de rendre compte d'une œuvre polémique, et celle de Gérard Chouquer n'échappe pas à la règle. Signe des temps, ce nouvel opus du pape de l'archéogéographie est dédié à une crise et non des moindres, celle des " récits géohistoriques", élaborés par les historiens et géographes historiques modernes sur des bases épistémologiques, dont les fondements relèvent depuis le XviI ${ }^{\mathrm{e}}$ siècle de la philosophie cartésienne puis de l'idéologie républicaine nationale. Entendons par là crise de l'Histoire-Géographie moderne, héritée en dernière instance des idoles $\mathrm{du} \mathrm{xx}^{\mathrm{e}}$ siècle (Marc Bloch, Fernand Braudel...), dans un contexte plus général et tout à fait d'actualité de crise des sciences humaines, ces sciences «molles » qui, par opposition à celles réputes "dures", subissent aujourd'hui les assauts d'une critique sans aucun doute nécessaire, mais souvent dirigée.

Dès la lecture du préambule, la composition et la finalité affichée de l'ouvrage suscitent une première interrogation : ce " traité " d'archéogéographie en est-il bien un? Le dialogue permanent entre la chose et le mot, l'objet et la forme, la question et les réponses composent un développement fourmillant, d'une grande richesse intellectuelle, soutenu par une érudition sans faille. Toutefois, la solution archéogéographique vers laquelle convergent l'ensemble de la démonstration et l'appareil critique ne nous apparaît pas d'une limpide évidence. À travers le prisme d'une critique érudite, souvent austère, émaillée de néologismes colorés (à connotation souvent « anatomique " et / ou " médicale ", tels ces « collecteurs hypertrophiés»), entre le je-ne-sais-quoi et le presque-rien de l'épistémologie contemporaine, s'esquisse un engagement individuel en faveur de l'intérêt collectif à travers la salutaire irruption du présent dans les sciences du passé. Il s'agirait donc davantage à nos yeux d'un essai voire d'un manifeste dont le contenu est avant tout question de regard, induit par les angles et focales retenus par l'auteur, de même que les mots soigneusement choisis et disséqués pour le dire. À ce titre, Gérard Chouquer et ses disciples archéogéographes font montre dans ces pages d'une maîtrise lexicologique remarquable, tout entière employée à la dissection d'un discours dominant dont ils démontent un par un les mécanismes culturels, politiques et idéologiques forgés par le nationalisme identitaire imputé (de façon peut-être un rien caricaturale?) aux « savants » des $\mathrm{XIX}^{\mathrm{e}}$ - $\mathrm{XX}^{\mathrm{e}}$ siècles.

L'exposé du " projet archéogéographique " se fonde sur trois enquêtes, dont l'enseignement est décliné au sein de deux parties. La première enquête est un constat, celui de «la modernité anachronique des objets prémodernes élaborés par les savants et donc de l'inadaptation de ces objets à l'étude des passés lointains ». Le terme " objet " est défini, p. 7, comme « des élaborations plus ou moins fondées, présentées comme des objets historiques avec lesquels on écrit d'ordinaire l'histoire de l'espace-temps " (domaine antique, villa, cité, centuriation, voie romaine, village, etc.). L'auteur propose donc une interrogation de la Modernité qui débouche sur une remise en cause, parfois radicale, des dits objets au regard des réalités qu'ils sont censés définir et condenser. La deuxième enquête aborde le problème de l'historiographie et en particulier celui du foisonnement disciplinaire qui la caractérise, signe d'un "individualisme méthodologique » que $\mathrm{G}$. Chouquer et $\mathrm{M}$. Watteaux dissèquent à travers pas moins d'une cinquantaine d'intitulés, disciplinaires ou thématiques. La troisième enquête propose enfin un cadre théorique et épistémologique à la rénovation des 
objets géohistoriques, envisageant de ce fait une véritable recomposition de la recherche autour de nouveaux objets qui sont ceux de l'archéogéographie. Cette entreprise s'entend comme "l'ébauche d'organisation consciente d'une représentation collective de la dynamique des espaces, représentation en partie héritée des disciplines existantes et en partie nouvelle et dont on verra qu'elle est à la fois cohérente et paradoxale ".

Dans la première partie de l'ouvrage, G. Chouquer s'intéresse à ce qu'il nomme les " collecteurs hypertrophiés " (p. 13 $s q$.), " représentations devenues méta-objets de science, mais dont l'ampleur même, accrue avec le temps, rend la définition quasi impossible et la pertinence discutable ». Sont alors successivement analysés les ethnotypes nationaux, la formation du territoire national, les fondements de la géographie historique française, le paysage, « l'espace-temps bivalent des utopies », la ville, la ruralité, la grande propriété antique, le patrimoine, le naturalisme et l'environnement, l'organisation sociale. Dans une seconde partie (p. 103 sq.), l'auteur analyse une dizaine d'" outils » élaborés par la Modernité en vue d'opérer la réduction des réalités objectives par effacement partiel de leur héritage : la cartographie, la métrologie, la géologie, la toponymie, la typologie agraire, la géométrie territoriale, l'archéologie (et sa " récupération par l'historicisme "), le "privilège exorbitant des objets romains ", la naturalisation des types architecturaux. En conclusion, l'accent est porté sur la remise en cause des " Caractères originaux » de Marc Bloch et les moyens d'une redéfinition de l'espace-temps moderne (p. 167 sq.).

Génial contestataire pour les uns, chantre irritant de la (ré) invention du fil à couper le beurre pour les autres, Gérard Chouquer nous offre un petit livre à la critique salutaire mais souvent déroutante, car en rupture avec les cheminements classiques (et confortables) de la pensée académique. À cet égard, cet essai s'inscrit bel et bien dans le droit fil de ses précédentes productions. La démarche ne manque pas de courage et pourrait même faire exemple dans le paysage actuel de la recherche archéologique, ne serait-ce que par la touchante générosité dont fait montre l'auteur envers ses élèves ou ses pairs. La lecture, quoi qu'il en soit, demeure ardue et n'est guère facilitée par les nombreuses coquilles émaillant le texte, qui se conclut par une bibliographie brutalement tronquée dont on aurait aimé connaître la fin, au prix modique d'une seule deux-centième page... Du point de vue des problèmes intellectuels qu'il soulève, cet ouvrage s'avère fort instructif et peut être recommandé à tous les archéologues concernés de près ou de loin par l'état de leur discipline. D'aucuns ne manqueront pas de s'interroger sur l'utilité finale de l'exercice : d'emblée, ceci figure dans le préambule. Ceux qui la réfutent s'abstiendront de lire cet ouvrage; les autres seront invités à reconsidérer sous un jour épistémologique les grands problèmes archéogéographiques de l'alignement des parcellaires, du Petit âge glaciaire, de l'encellulement médiéval, de la tectonique des plaques réunies au sein d'une même et vaste fratrie : celle des " formes " élaborées par le jeu simultané de la nature et des sociétés. La question principale, fil directeur du raisonnement, est la suivante : comment passer de la forme, du fait, à l'objet historique (p. 6-7)? Question déjà ancienne, dont est d'ailleurs rappelé l'arrière-plan philosophique (Emmanuel Kant et les philosophes allemands de la Gellstat, Archéologie du savoir de Michel Foucault...). L'auteur s'attache ainsi à entériner plus qu'à opérer la destruction des concepts ou « méta-objets » véhiculés par l'histoire-géographie académique $\mathrm{du} \mathrm{xx}^{\mathrm{e}}$ siècle, en vue de leur substituer les nouveaux objets-objectifs de l'archéogéographie spatialiste. De fait, une question finale s'impose, à laquelle nous ne saurions, justement, apporter de réponse objective : sommes-nous bien confrontés ici à une rénovation proprement dite des objets " en crise "? et, dans l'affirmative, combien durable et au profit de quelle école? Le lecteur tranchera.

Vincent CARPENTIER INRAP/Centre Michel de Boüard-CRAHAM, FRE 3119 